\title{
Some inequalities for the Hadamard product of an $M$-matrix and an inverse $M$-matrix
}

\author{
Duanmei Zhou', Guoliang Chen ${ }^{1 *}$, Guoxing $\mathrm{Wu}^{2}$ and Xiangyun Zhang ${ }^{1}$
}

${ }^{\text {*Correspondence: }}$

glchen@math.ecnu.edu.cn

1 Department of Mathematics, East China Normal University, Shanghai, 200241, People's Republic of China Full list of author information is available at the end of the article

\begin{abstract}
Let $A$ and $B$ be nonsingular $M$-matrices. Some new lower bounds on the minimum eigenvalue $q\left(A \circ B^{-1}\right)$ for the Hadamard product of $A$ and $B^{-1}$ are given. These bounds improve the corresponding results of Chen (Linear Algebra Appl. 378:159-166, 2004) and Huang (Linear Algebra Appl. 428:1551-1559, 2008) and generalize the corresponding result of Xiang (Linear Algebra Appl. 367:17-27, 2003).

MSC: $15 \mathrm{~A} 06 ; 15 \mathrm{~A} 15 ; 15 \mathrm{~A} 48$
\end{abstract}

Keywords: M-matrix; Hadamard product; minimum eigenvalue

\section{Introduction}

For a positive integer $n, N$ denotes the set $\{1,2, \ldots, n\}$. The set of all $n \times n$ complex matrices is denoted by $\mathbb{C}^{n \times n}$, and $\mathbb{R}^{n \times n}$ denotes the set of all $n \times n$ real matrices throughout.

Let $A=\left(a_{i j}\right) \in \mathbb{R}^{n \times n}$ and $B=\left(b_{i j}\right) \in \mathbb{R}^{n \times n}$. We write $A \geq B(>B)$ if $a_{i j} \geq b_{i j}\left(>b_{i j}\right)$ for all $1 \leq i \leq n, 1 \leq j \leq n$. If $A \geq 0(>0)$, we say that $A$ is a nonnegative (positive) matrix. The spectral radius of $A$ is denoted by $\rho(A)$. Let $A$ be an irreducible nonnegative matrix. It is well known that there exists a positive vector $u$ such that $A u=\rho(A) u, u$ being called a right Perron eigenvector of $A$. This guarantees that $\rho(A) \in \sigma(A)$, where $\sigma(A)$ denotes the spectrum of $A$.

The set $Z_{n} \subset \mathbb{R}^{n \times n}$ is defined by

$$
Z_{n} \equiv\left\{A=\left(a_{i j}\right) \in \mathbb{R}^{n \times n}: a_{i j} \leq 0 \text { if } i \neq j, i, j=1, \ldots, n\right\} .
$$

The simple sign patten of the matrices in $Z_{n}$ has many striking consequences. Let $A=$ $\left(a_{i j}\right) \in Z_{n}$ and suppose $A=\alpha I-P$ with $\alpha \in \mathbb{R}$ and $P \geq 0$. Then $\alpha-\rho(P)$ is an eigenvalue of $A$, every eigenvalue of $A$ lies in the disc $\{z \in \mathbb{C}:|z-\alpha| \leq \rho(P)\}$, and hence every eigenvalue $\lambda$ of $A$ satisfies $\operatorname{Re} \lambda \geq \alpha-\rho(P)$. In particular, a matrix $A \in Z_{n}$ is called an $M$-matrix if $\alpha \geq \rho(P)$. If $\alpha>\rho(P)$, we call $A$ nonsingular $M$-matrix, and denote the class of nonsingular $M$-matrices by $M_{n}$.

Let $A=\left(a_{i j}\right) \in Z_{n}$, we denote $\min \{\operatorname{Re}(\lambda): \lambda \in \sigma(A)\}$ by $q(A)$. The following simple facts are needed for our purpose in proving (see Problems 16 and 19 in Section 2.5 of [1]):

(i) $q(A) \in \sigma(A) ; q(A)$ is called a minimum eigenvalue of $A$.

(ii) If $A \in M_{n}$ and $\rho\left(A^{-1}\right)$ is the Perron eigenvalue of the nonnegative matrix $A^{-1}$, then $q(A)=\frac{1}{\rho\left(A^{-1}\right)}$ is a positive real eigenvalue of $A$.

Let $A$ be an irreducible nonsingular $M$-matrix. It is well known that there exists a positive vector $u$ such that $A u=q(A) u, u$ being called a right Perron eigenvector of $A$.

(c) 2013 Zhou et al.; licensee Springer. This is an Open Access article distributed under the terms of the Creative Commons Attribution License (http://creativecommons.org/licenses/by/2.0), which permits unrestricted use, distribution, and reproduction in any medium, provided the original work is properly cited. 
If $A=\left(a_{i j}\right) \in M_{n}$, we write $C_{A}=D_{A}-A$, where $D_{A}=\operatorname{diag}\left(a_{i i}\right)$. Note that $a_{i i}>0$ for all $i \in N$ if $A \in M_{n}$. Thus we define the Jacobi iterative matrix of $A$ by $J_{A}=D_{A}^{-1} C_{A}$. It is easy to check that $J_{A}$ is nonnegative and $\rho\left(J_{A}\right)<1$ (see [2]).

The Hadamard product of $A=\left(a_{i j}\right) \in \mathbb{C}^{n \times n}$ and $B=\left(b_{i j}\right) \in \mathbb{C}^{n \times n}$ is defined by $A \circ B \equiv$ $\left(a_{i j} b_{i j}\right) \in \mathbb{C}^{n \times n}$.

It has been noted $[3,4]$ that the Hadamard product $B \circ A^{-1}$ of an $M$-matrix $B$ and the inverse of an $M$-matrix $A$ is again an $M$-matrix.

In 1991, Horn et al. [1, p. 375] showed the classical result: if $A=\left(a_{i j}\right) \in M_{n}, B=\left(b_{i j}\right) \in M_{n}$, $B^{-1}=\left(\beta_{i j}\right)$, then

$$
q\left(A \circ B^{-1}\right) \geq q(A) \min _{1 \leq i \leq n} \beta_{i i}
$$

Subsequently, Chen [5] improved the bound in (1.1) and obtained the following result:

$$
q\left(A \circ B^{-1}\right) \geq q(A) q(B) \min _{1 \leq i \leq n}\left\{\left(\frac{a_{i i}}{q(A)}+\frac{b_{i i}}{q(B)}-1\right) \frac{\beta_{i i}}{b_{i i}}\right\} .
$$

In 2008, Huang [2] obtained the following result:

$$
q\left(A \circ B^{-1}\right) \geq \frac{1-\rho\left(J_{A}\right) \rho\left(J_{B}\right)}{1+\rho^{2}\left(J_{B}\right)} \min _{1 \leq i \leq n} \frac{a_{i i}}{b_{i i}} .
$$

This bound in (1.3) improved the bound in (1.1) in some cases. For example, if

$$
B=\left(\begin{array}{ll}
4 & 0 \\
0 & 3
\end{array}\right), \quad A=\left(\begin{array}{cc}
3 & -1 \\
0 & 2
\end{array}\right)
$$

then $q\left(A \circ B^{-1}\right)=\frac{1-\rho\left(J_{A}\right) \rho\left(J_{B}\right)}{1+\rho^{2}\left(J_{B}\right)} \min _{1 \leq i \leq n} \frac{a_{i i}}{b_{i i}}=\frac{2}{3} \geq q(A) \min _{1 \leq i \leq n} \beta_{i i}=\frac{1}{2}$. But $\frac{1-\rho\left(J_{A}\right) \rho\left(J_{B}\right)}{1+\rho^{2}\left(J_{B}\right)} \times$ $\min _{1 \leq i \leq n} \frac{a_{i i}}{b_{i i}} \leq q(A) \min _{1 \leq i \leq n} \beta_{i i}$ in Example 2.1 in this paper.

In practice, the bound of $q\left(A \circ B^{-1}\right)$ can give a rough estimate before actually solving it and can serve as a check of whether the solution technique for it actually resulted in valid solution. Besides, a good bound of $q\left(A \circ B^{-1}\right)$ can also help us reduce the computational burden. Therefore, it is necessary to study the bound. In this paper, we present some new lower bounds of the minimum eigenvalue $q\left(A \circ B^{-1}\right)$ for the Hadamard product of $M$ matrices, which improve (1.1), (1.2) and (1.3) and generalize the corresponding result of Xiang [6].

\section{Main results}

In this section, we state and prove our main results. Firstly, we give some lemmas.

Lemma 2.1 (See [7, Theorem 11]) Let $A=\left(a_{i j}\right) \in \mathbb{C}^{n \times n}$, with $n \geq 2$. Then, if $\lambda$ is an eigenvalue of $A$, there is a pair $(r, q)$ of positive integers with $r \neq q(1 \leq r, q \leq n)$ such that

$$
\left|\lambda-a_{r r}\right| \cdot\left|\lambda-a_{q q}\right| \leq \sum_{k \neq r}\left|a_{r k}\right| \cdot \sum_{l \neq q}\left|a_{q l}\right| .
$$


Lemma 2.2 (See [8, Lemma 2.2]) (a) If $A=\left(a_{i j}\right)$ is an $n \times n$ strictly diagonally dominant matrix by row, that is, $\left|a_{i i}\right|>\sum_{j \neq i}\left|a_{i j}\right|$ for any $i \in N$, then $A^{-1}=\left(\beta_{i j}\right)$ exists, and

$$
\left|\beta_{j i}\right| \leq \frac{\sum_{k \neq j}\left|a_{j k}\right|}{\left|a_{j j}\right|}\left|\beta_{i i}\right|, \quad \text { for all } j \neq i
$$

(b) If $A=\left(a_{i j}\right)$ is an $n \times n$ strictly diagonally dominant matrix by column, that is, $\left|a_{i i}\right|>$ $\sum_{j \neq i}\left|a_{j i}\right|$ for any $i \in N$, then $A^{-1}=\left(\beta_{i j}\right)$ exists, and

$$
\left|\beta_{i j}\right| \leq \frac{\sum_{k \neq j}\left|a_{k j}\right|}{\left|a_{j j}\right|}\left|\beta_{i i}\right|, \quad \text { for all } j \neq i
$$

Proof We give a simple proof of (a) which is different from that in [8]. Similarly, one can prove (b). Firstly, we prove $\left|\beta_{j i}\right| \leq\left|\beta_{i i}\right|$ for all $j \neq i$. Suppose not. Let $\left|\beta_{j i}\right| \geq\left|\beta_{i i}\right|$ for some $j$ and $j \neq i$. We can then assume $\left|\beta_{j i}\right| \geq\left|\beta_{k i}\right|$ for all $k \in N$. Since $A A^{-1}=I$, we have $\sum_{k=1}^{n} a_{j k} \beta_{k i}=0$. Thus

$$
\left|a_{j j} \beta_{j i}\right| \leq \sum_{k \neq j}\left|a_{j k} \beta_{k i}\right| \leq \sum_{k \neq j}\left|a_{j k}\right|\left|\beta_{j i}\right|<\left|a_{j j}\right|\left|\beta_{j i}\right|,
$$

which is a contradiction. Hence, $\left|\beta_{j i}\right| \leq\left|\beta_{i i}\right|$ holds for all pairs $i, j$. Thus

$$
\left|a_{j j} \beta_{j i}\right| \leq \sum_{k \neq j}\left|a_{j k} \beta_{k i}\right| \leq \sum_{k \neq j}\left|a_{j k}\right|\left|\beta_{i i}\right|, \quad \text { for all } j \neq i
$$

that is,

$$
\left|\beta_{j i}\right| \leq \frac{\sum_{k \neq j}\left|a_{j k}\right|}{\left|a_{j j}\right|}\left|\beta_{i i}\right|, \quad \text { for all } j \neq i
$$

Theorem 2.3 Let $A=\left(a_{i j}\right) \in M_{n}, B=\left(b_{i j}\right) \in M_{n}$ and $B^{-1}=\left(\beta_{i j}\right)$. Then

$$
\begin{aligned}
q\left(A \circ B^{-1}\right) \geq & \min _{i \neq j} \frac{1}{2}\left\{a_{i i} \beta_{i i}+a_{j j} \beta_{j j}-\left[\left(a_{i i} \beta_{i i}-a_{i j} \beta_{j j}\right)^{2}\right.\right. \\
& \left.\left.+4 \frac{\beta_{i i} \beta_{j j}}{b_{i i} b_{j j}}\left[b_{i i}-q(B)\right]\left[a_{i i}-q(A)\right]\left[b_{j j}-q(B)\right]\left[a_{j j}-q(A)\right]\right]^{\frac{1}{2}}\right\} .
\end{aligned}
$$

Proof If both $A$ and $B$ are irreducible. Let $v=\left(v_{i}\right)$ and $y=\left(y_{i}\right)$ be the right Perron eigenvectors of $B^{T}$ and $A$, respectively, i.e., $B^{T} v=q\left(B^{T}\right) v=q(B) v, A y=q(A) y$. Define $C=B^{T} V$, where $V=\operatorname{diag}\left(v_{1}, v_{2}, \ldots, v_{n}\right)$. It is easy to check that $C$ is diagonally dominant by row. It follows from Lemma 2.2 , for all $i \neq j$, we have

$$
\frac{\beta_{i j}}{v_{j}} \leq \frac{\sum_{k \neq j}\left|v_{k} b_{k j}\right|}{v_{j} b_{j j}} \frac{\beta_{i i}}{v_{i}}=\frac{\left(b_{j j}-q(B)\right) v_{j}}{b_{j j} v_{j}} \frac{\beta_{i i}}{v_{i}} .
$$

Thus

$$
\beta_{i j} \leq \frac{\left(b_{j j}-q(B)\right) v_{j} \beta_{i i}}{b_{j j} v_{i}}
$$


Let $s_{j}=\frac{\left(b_{j j}-q(B)\right) v_{j}}{b_{j j} y_{j}}, S=\operatorname{diag}\left(s_{1}, s_{2}, \ldots, s_{n}\right)$. Then $S>0$ and

$$
S\left(A \circ B^{-1}\right) S^{-1}=\left(\begin{array}{cccc}
a_{11} \beta_{11} & s_{1} a_{12} \beta_{12} / s_{2} & \cdots & s_{1} a_{1 n} \beta_{1 n} / s_{n} \\
s_{2} a_{21} \beta_{21} / s_{1} & a_{22} \beta_{22} & \cdots & s_{2} a_{2 n} \beta_{2 n} / s_{n} \\
\vdots & \vdots & \ddots & \vdots \\
s_{n} a_{n 1} \beta_{n 1} / s_{1} & s_{n} a_{n 2} \beta_{n 2} / s_{2} & \cdots & a_{n n} \beta_{n n}
\end{array}\right) .
$$

Hence, $\sigma\left(A \circ B^{-1}\right)=\sigma\left(S\left(A \circ B^{-1}\right) S^{-1}\right)$. Since $q\left(A \circ B^{-1}\right)$ is an eigenvalue of $A \circ B^{-1}$, we have

$$
q\left(A \circ B^{-1}\right) \in \sigma\left(S\left(A \circ B^{-1}\right) S^{-1}\right) .
$$

Thus, by Lemma 2.1, there exists a pair $(i, j)$ of positive integers with $i \neq j(1 \leq i, j \leq n)$ such that

$$
\begin{aligned}
& \left|q\left(A \circ B^{-1}\right)-a_{i i} \beta_{i i}\right|\left|q\left(A \circ B^{-1}\right)-a_{j j} \beta_{j j}\right| \\
& \leq \sum_{k \neq i} \frac{\left|a_{i k} \beta_{i k}\right|}{s_{k}} s_{i} \sum_{l \neq j} \frac{\left|a_{j l} \beta_{j l}\right|}{s_{l}} s_{j} \\
& \leq s_{i} \sum_{k \neq i} \frac{\left|a_{i k}\right|\left(b_{k k}-q(B)\right) v_{k} \beta_{i i}}{b_{k k} v_{i}} \frac{b_{k k} y_{k}}{\left(b_{k k}-q(B)\right) v_{k}} s_{j} \sum_{l \neq j} \frac{\left|a_{j l}\right|\left(b_{l l}-q(B)\right) v_{l} \beta_{j j}}{b_{l l} v_{j}} \frac{b_{l l} y_{l}}{\left(b_{l l}-q(B)\right) v_{l}} \\
& =s_{i} \frac{\beta_{i i}}{v_{i}} \sum_{k \neq i}\left|a_{i k}\right| y_{k} s_{j} \frac{\beta_{j j}}{v_{j}} \sum_{l \neq j}\left|a_{j l}\right| y_{l} \\
& =\frac{\left(b_{i i}-q(B)\right) v_{i}}{b_{i i} y_{i}} \frac{\beta_{i i}}{v_{i}}\left(a_{i i}-q(A)\right) y_{i} \frac{\left(b_{j j}-q(B)\right) v_{j}}{b_{j j} y_{j}} \frac{\beta_{j j}}{v_{j}}\left(a_{j j}-q(A)\right) y_{j} \\
& =\frac{\beta_{i i} \beta_{j j}}{b_{i i} b_{j j}}\left[b_{i i}-q(B)\right]\left[a_{i i}-q(A)\right]\left[b_{j j}-q(B)\right]\left[a_{j j}-q(A)\right] .
\end{aligned}
$$

From the above inequality and $0 \leq q\left(A \circ B^{-1}\right) \leq a_{i i} \beta_{i i}, \forall i \in N$, we have

$$
\begin{aligned}
& \left(q\left(A \circ B^{-1}\right)-a_{i i} \beta_{i i}\right)\left(q\left(A \circ B^{-1}\right)-a_{j j} \beta_{j j}\right) \\
& \quad \leq \frac{\beta_{i i} \beta_{j j}}{b_{i i} b_{j j}}\left[b_{i i}-q(B)\right]\left[a_{i i}-q(A)\right]\left[b_{j j}-q(B)\right]\left[a_{j j}-q(A)\right] .
\end{aligned}
$$

Thus, from inequality (2.2), we have

$$
\begin{aligned}
q\left(A \circ B^{-1}\right) \geq & \frac{1}{2}\left\{a_{i i} \beta_{i i}+a_{j j} \beta_{j j}-\left[\left(a_{i i} \beta_{i i}-a_{j j} \beta_{j j}\right)^{2}\right.\right. \\
& \left.\left.+4 \frac{\beta_{i i} \beta_{j j}}{b_{i i} b_{j j}}\left[b_{i i}-q(B)\right]\left[a_{i i}-q(A)\right]\left[b_{j j}-q(B)\right]\left[a_{j j}-q(A)\right]\right]^{\frac{1}{2}}\right\} \\
\geq & \min _{i \neq j} \frac{1}{2}\left\{a_{i i} \beta_{i i}+a_{j j} \beta_{j j}-\left[\left(a_{i i} \beta_{i i}-a_{j j} \beta_{j j}\right)^{2}\right.\right. \\
& \left.\left.+4 \frac{\beta_{i i} \beta_{j j}}{b_{i i} b_{j j}}\left[b_{i i}-q(B)\right]\left[a_{i i}-q(A)\right]\left[b_{i j}-q(B)\right]\left[a_{j j}-q(A)\right]\right]^{\frac{1}{2}}\right\} .
\end{aligned}
$$


Assume that one of $A$ and $B$ is reducible. It is well known that a matrix in $Z_{n}$ is a nonsingular $M$-matrix if and only if all its leading principal minors are positive (see condition (E17) of Theorem 6.2.3 of [9]). If we denote by $D=\left(d_{i j}\right)$ the $n \times n$ permutation matrix with $d_{12}=d_{23}=\cdots=d_{n-1 n}=d_{n 1}=1$, the remaining $d_{i j}$ zero, then both $A-t D$ and $B-t D$ are irreducible nonsingular $M$-matrices for any chosen positive real number $t$ sufficiently small such that all the leading principal minors of both $A-t D$ and $B-t D$ are positive. Now, we substitute $A-t D$ and $B-t D$ for $A$ and $B$, respectively, in the previous case, and then letting $t \rightarrow 0$, the result follows by continuity.

Using ideas of the proof of Theorem 2.3, we next give a new proof of inequality (2.2) in [5]. Similar to the proof of Theorem 2.3, by the theorem of Gerschgorin, there exist some positive integers $i \in N$ such that

$$
\begin{aligned}
\left|q\left(A \circ B^{-1}\right)-a_{i i} \beta_{i i}\right| & \leq \sum_{k \neq i} \frac{\left|a_{i k} \beta_{i k}\right|}{s_{k}} s_{i} \\
& \leq s_{i} \sum_{k \neq i} \frac{\left|a_{i k}\right|\left(b_{k k}-q(B)\right) v_{k} \beta_{i i}}{b_{k k} v_{i}} \frac{b_{k k} y_{k}}{\left(b_{k k}-q(B)\right) v_{k}} \\
& =s_{i} \frac{\beta_{i i}}{v_{i}} \sum_{k \neq i}\left|a_{i k}\right| y_{k} \\
& =\frac{\left(b_{i i}-q(B)\right) v_{i}}{b_{i i} y_{i}} \frac{\beta_{i i}}{v_{i}}\left(a_{i i}-q(A)\right) y_{i} \\
& =\frac{\beta_{i i}}{b_{i i}}\left[b_{i i}-q(B)\right]\left[a_{i i}-q(A)\right] .
\end{aligned}
$$

From the above inequality and $0 \leq q\left(A \circ B^{-1}\right) \leq a_{i i} \beta_{i i}, \forall i \in N$, we have

$$
a_{i i} \beta_{i i}-q\left(A \circ B^{-1}\right) \leq \frac{\beta_{i i}}{b_{i i}}\left[b_{i i}-q(B)\right]\left[a_{i i}-q(A)\right] .
$$

Thus, from inequality (2.3), we have

$$
\begin{aligned}
q\left(A \circ B^{-1}\right) & \geq a_{i i} \beta_{i i}-\frac{\beta_{i i}}{b_{i i}}\left[b_{i i}-q(B)\right]\left[a_{i i}-q(A)\right] \\
& =q(A) q(B)\left\{\left(\frac{a_{i i}}{q(A)}+\frac{b_{i i}}{q(B)}-1\right) \frac{\beta_{i i}}{b_{i i}}\right\} \\
& \geq q(A) q(B) \min _{1 \leq i \leq n}\left\{\left(\frac{a_{i i}}{q(A)}+\frac{b_{i i}}{q(B)}-1\right) \frac{\beta_{i i}}{b_{i i}}\right\} .
\end{aligned}
$$

Remark 2.1 We next give a simple comparison between the upper bound in (2.1) and the upper bound in (1.2) and (1.1). Without loss of generality, for $i \neq j$, assume that

$$
a_{i i} \beta_{i i}-\frac{\beta_{i i}}{b_{i i}}\left[b_{i i}-q(B)\right]\left[a_{i i}-q(A)\right] \leq a_{i j} \beta_{j j}-\frac{\beta_{j j}}{b_{j j}}\left[b_{j j}-q(B)\right]\left[a_{j j}-q(A)\right] .
$$

Thus, we can write (2.4) equivalently as

$$
\frac{\beta_{j j}}{b_{j j}}\left[b_{j j}-q(B)\right]\left[a_{i j}-q(A)\right] \leq a_{j j} \beta_{i j}-a_{i i} \beta_{i i}+\frac{\beta_{i i}}{b_{i i}}\left[b_{i i}-q(B)\right]\left[a_{i i}-q(A)\right] .
$$


From (2.1), we have

$$
\begin{gathered}
\left(a_{i i} \beta_{i i}-a_{j j} \beta_{j j}\right)^{2}+4 \frac{\beta_{i i} \beta_{j j}}{b_{i i} b_{j j}}\left[b_{i i}-q(B)\right]\left[a_{i i}-q(A)\right]\left[b_{j j}-q(B)\right]\left[a_{j j}-q(A)\right] \\
\leq\left(a_{j j} \beta_{j j}-a_{i i} \beta_{i i}\right)^{2}+4 \frac{\beta_{i i}}{b_{i i}}\left[b_{i i}-q(B)\right]\left[a_{i i}-q(A)\right]\left(a_{j j} \beta_{j j}-a_{i i} \beta_{i i}\right) \\
\quad+4\left[\frac{\beta_{i i}}{b_{i i}}\left[b_{i i}-q(B)\right]\left[a_{i i}-q(A)\right]\right]^{2} \\
=\left(a_{j j} \beta_{j j}-a_{i i} \beta_{i i}+2 \frac{\beta_{i i}}{b_{i i}}\left[b_{i i}-q(B)\right]\left[a_{i i}-q(A)\right]\right)^{2} .
\end{gathered}
$$

Thus, from (2.1), (2.5) and the above inequality, we have

$$
\begin{aligned}
q\left(A \circ B^{-1}\right) \geq & \min _{i \neq j} \frac{1}{2}\left\{a_{i i} \beta_{i i}+a_{j j} \beta_{j j}-\left[\left(a_{i i} \beta_{i i}-a_{j j} \beta_{j j}\right)^{2}\right.\right. \\
& \left.\left.+4 \frac{\beta_{i i} \beta_{j j}}{b_{i i} b_{j j}}\left[b_{i i}-q(B)\right]\left[a_{i i}-q(A)\right]\left[b_{j j}-q(B)\right]\left[a_{j j}-q(A)\right]\right]^{\frac{1}{2}}\right\} \\
\geq & \min _{i \neq j} \frac{1}{2}\left\{a_{i i} \beta_{i i}+a_{j j} \beta_{j j}-a_{j j} \beta_{j j}+a_{i i} \beta_{i i}-2 \frac{\beta_{i i}}{b_{i i}}\left[b_{i i}-q(B)\right]\left[a_{i i}-q(A)\right]\right\} \\
\geq & q(A) q(B) \min _{1 \leq i \leq n}\left\{\left(\frac{a_{i i}}{q(A)}+\frac{b_{i i}}{q(B)}-1\right) \frac{\beta_{i i}}{b_{i i}}\right\} .
\end{aligned}
$$

Hence, the bound in (2.1) is sharper than the bound in (1.2). According to Remark 2.4 in [5], we know

$$
q(A) q(B) \min _{1 \leq i \leq n}\left\{\left(\frac{a_{i i}}{q(A)}+\frac{b_{i i}}{q(B)}-1\right) \frac{\beta_{i i}}{b_{i i}}\right\} \geq q(A) \min _{1 \leq i \leq n} \beta_{i i} .
$$

So, the bound in (2.1) is sharper than the bound in (1.1).

Theorem 2.4 Let $A=\left(a_{i j}\right) \in M_{n}, B=\left(b_{i j}\right) \in M_{n}$. Then

$$
q\left(A \circ B^{-1}\right) \geq\left(1-\rho\left(J_{A}\right) \rho\left(J_{B}\right)\right) \min _{1 \leq i \leq n} \frac{a_{i i}}{b_{i i}} .
$$

Proof Suppose that $A$ and $B$ are irreducible, $D_{B}$ is the diagonal matrix of $B$ and $C_{B}=D_{B}-B$, then $D_{B}$ is a diagonal matrix with positive diagonal entries, $C_{B}$ is an irreducible nonnegative matrix and $J=D_{B}^{-1} C_{B}^{T}$ is again an irreducible nonnegative matrix. Since the Jacobi iterative matrix of $B$ is $J_{B}=D_{B}^{-1} C_{B}$, we have

$$
\rho\left(J_{B}\right)=\rho\left(D_{B}^{-1} C_{B}\right)=\rho\left(\left(D_{B}^{-1} C_{B}\right)^{T}\right)=\rho\left(C_{B}^{T} D_{B}^{-1}\right)=\rho\left(D_{B}^{-1} C_{B}^{T}\right)=\rho(J) .
$$

By the Perron-Frobenius theorem on irreducible nonnegative matrices, there is a positive eigenvector $x=\left(x_{1}, x_{2}, \ldots, x_{n}\right)^{T}$ such that $D_{B}^{-1} C_{B}^{T} x=\rho(J) x$. That is,

$$
\sum_{k \neq i} \frac{\left|b_{k i}\right| x_{k}}{b_{i i}}=\rho(J) x_{i} \quad \forall i \in N
$$


Thus, we can write (2.8) equivalently as

$$
\sum_{k \neq i} \frac{\left|b_{k i}\right| x_{k}}{b_{i i} x_{i}}=\rho(J) \quad \forall i \in N
$$

Set $X=\operatorname{diag}\left(x_{1}, x_{2}, \ldots, x_{n}\right)$ and $\widetilde{B}=X B$. It is easy to check that $\widetilde{B}$ is a strictly diagonally dominant matrix by column. Let $B^{-1}=\left(\beta_{i j}\right)$. By Lemma 2.2 , for all $i \neq j(1 \leq i, j \leq n)$, we have

$$
\beta_{i j} x_{j}^{-1} \leq \frac{\sum_{k \neq j}\left|b_{k j}\right| x_{k}}{b_{j j} x_{j}} \beta_{i i} x_{i}^{-1}=\rho(J) \beta_{i i} x_{i}^{-1} .
$$

Thus

$$
\beta_{i j} \leq \rho(J) \beta_{i i} \frac{x_{j}}{x_{i}} \quad \forall i \in N
$$

Combining (2.9) with (2.7), we get

$$
\beta_{i j} \leq \rho\left(J_{B}\right) \beta_{i i} \frac{x_{j}}{x_{i}}
$$

Since $B^{-1} B=I$, we obtain

$$
\beta_{i i} b_{i i}=1+\sum_{k \neq i} \beta_{i k}\left|b_{k i}\right| \geq 1 \quad \forall i \in N .
$$

Thus

$$
\beta_{i i} \geq \frac{1}{b_{i i}} \quad \forall i \in N
$$

Let $J_{A} y=\rho\left(J_{A}\right) y$ for positive vectors $y=\left(y_{i}\right)$. Set $S=\operatorname{diag}\left(\frac{x_{1}}{y_{1}}, \frac{x_{2}}{y_{2}}, \ldots, \frac{x_{n}}{y_{n}}\right)$, then $S>0$. Hence, $\sigma\left(A \circ B^{-1}\right)=\sigma\left(S\left(A \circ B^{-1}\right) S^{-1}\right)$. Since $q\left(A \circ B^{-1}\right)$ is an eigenvalue of $A \circ B^{-1}$, we have

$$
q\left(A \circ B^{-1}\right) \in \sigma\left(S\left(A \circ B^{-1}\right) S^{-1}\right)
$$

By the theorem of Gerschgorin and (2.10), there exist some positive integers $i \in N$ such that

$$
\begin{aligned}
\left|q\left(A \circ B^{-1}\right)-a_{i i} \beta_{i i}\right| & \leq \sum_{k \neq i}\left|a_{i k} \beta_{i k}\right| \frac{x_{i} y_{k}}{y_{i} x_{k}} \\
& \leq \frac{x_{i}}{y_{i}} \sum_{k \neq i}\left|a_{i k}\right| \rho\left(J_{B}\right) \beta_{i i} \frac{x_{k}}{x_{i}} \frac{y_{k}}{x_{k}} \\
& =\rho\left(J_{B}\right) \frac{\beta_{i i}}{y_{i}} \sum_{k \neq i}\left|a_{i k}\right| y_{k} \\
& =a_{i i} \beta_{i i} \rho\left(J_{A}\right) \rho\left(J_{B}\right) .
\end{aligned}
$$


From the above inequality and $0 \leq q\left(A \circ B^{-1}\right) \leq a_{i i} \beta_{i i}, \forall i \in N$, we have

$$
a_{i i} \beta_{i i}-q\left(A \circ B^{-1}\right) \leq a_{i i} \beta_{i i} \rho\left(J_{A}\right) \rho\left(J_{B}\right)
$$

Thus, from inequality (2.11) and (2.12), we have

$$
\begin{aligned}
q\left(A \circ B^{-1}\right) & \geq a_{i i} \beta_{i i}-a_{i i} \beta_{i i} \rho\left(J_{A}\right) \rho\left(J_{B}\right) \\
& =\left(1-\rho\left(J_{A}\right) \rho\left(J_{B}\right)\right) a_{i i} \beta_{i i} \\
& \geq\left(1-\rho\left(J_{A}\right) \rho\left(J_{B}\right)\right) \frac{a_{i i}}{b_{i i}} \\
& \geq\left(1-\rho\left(J_{A}\right) \rho\left(J_{B}\right)\right) \min _{1 \leq i \leq n} \frac{a_{i i}}{b_{i i}} .
\end{aligned}
$$

Assume that one of $A$ and $B$ is reducible. It is well known that a matrix in $Z_{n}$ is a nonsingular $M$-matrix if and only if all its leading principal minors are positive (see condition (E17) of Theorem 6.2.3 of [9]). If we denote by $D=\left(d_{i j}\right)$ the $n \times n$ permutation matrix with $d_{12}=d_{23}=\cdots=d_{n-1 n}=d_{n 1}=1$, the remaining $d_{i j}$ zero, then both $A-t D$ and $B-t D$ are irreducible nonsingular $M$-matrices for any chosen positive real number $t$ sufficiently small such that all the leading principal minors of both $A-t D$ and $B-t D$ are positive. Now, we substitute $A-t D$ and $B-t D$ for $A$ and $B$, respectively, in the previous case, and then letting $t \rightarrow 0$, the result follows by continuity.

Remark 2.2 If $B \in M_{n}$ is a diagonal matrix, the equality of (2.6) holds. Thus the bound (2.6) is sharp. Since $1+\rho^{2}\left(J_{B}\right) \geq 1$, then

$$
\left(1-\rho\left(J_{A}\right) \rho\left(J_{B}\right)\right) \min _{1 \leq i \leq n} \frac{a_{i i}}{b_{i i}} \geq \frac{1-\rho\left(J_{A}\right) \rho\left(J_{B}\right)}{1+\rho^{2}\left(J_{B}\right)} \min _{1 \leq i \leq n} \frac{a_{i i}}{b_{i i}} .
$$

The bound in (2.6) is sharper than the bound in (1.3).

If $B=A$, according to Theorem 2.4 , we can deduce the following corollary.

Corollary 2.5 Let $B \in M_{n}$, then

$$
q\left(B \circ B^{-1}\right) \geq 1-\rho^{2}\left(J_{B}\right)
$$

Remark 2.3 Corollary 2.5 is Theorem 2.8 of Xiang [6]. So, Theorem 2.4 generalizes Theorem 2.8 in [6].

If we apply Lemma 2.1 to $J=D_{B}^{-1} C_{B}^{T}$ and $J_{B}=D_{B}^{-1} C_{B}$, then we have

$$
\begin{aligned}
& \rho^{2}(J) \leq \max _{i \neq j} \sum_{k \neq i} \frac{\left|b_{k i}\right|}{b_{i i}} \cdot \sum_{l \neq j} \frac{\left|b_{l j}\right|}{b_{j j}}, \\
& \rho^{2}\left(J_{B}\right) \leq \max _{i \neq j} \sum_{k \neq i} \frac{\left|b_{i k}\right|}{b_{i i}} \cdot \sum_{l \neq j} \frac{\left|b_{j l}\right|}{b_{j j}} .
\end{aligned}
$$


Since $\rho\left(J_{B}\right)=\rho(J)$, then

$$
\rho^{2}\left(J_{B}\right) \leq \min \left\{\max _{i \neq j} \sum_{k \neq i} \frac{\left|b_{i k}\right|}{b_{i i}} \cdot \sum_{l \neq j} \frac{\left|b_{j l}\right|}{b_{j j}}, \max _{i \neq j} \sum_{k \neq i} \frac{\left|b_{k i}\right|}{b_{i i}} \cdot \sum_{l \neq j} \frac{\left|b_{l j}\right|}{b_{j j}}\right\} .
$$

From (2.13) we have the following corollary.

Corollary 2.6 Let $B=\left(b_{i j}\right) \in M_{n}$, then

$$
q\left(B \circ B^{-1}\right) \geq 1-\min \left\{\max _{i \neq j} \sum_{k \neq i} \frac{\left|b_{i k}\right|}{b_{i i}} \cdot \sum_{l \neq j} \frac{\left|b_{j l}\right|}{b_{j j}}, \max _{i \neq j} \sum_{k \neq i} \frac{\left|b_{k i}\right|}{b_{i i}} \cdot \sum_{l \neq j} \frac{\left|b_{l j}\right|}{b_{j j}}\right\} .
$$

Example 2.1 Let $A$ and $B$ be the same as in Example 2.1 in [10]:

$$
B=\left(\begin{array}{cccc}
4 & -1 & -1 & -1 \\
-2 & 5 & -1 & -1 \\
0 & -2 & 4 & -1 \\
-1 & -1 & -1 & 4
\end{array}\right), \quad A=\left(\begin{array}{cccc}
1 & -1 / 2 & 0 & 0 \\
-1 / 2 & 1 & -1 / 2 & 0 \\
0 & -1 / 2 & 1 & -1 / 2 \\
0 & 0 & -1 / 2 & 1
\end{array}\right)
$$

It is easy to check that $A, B \in M_{4}$. If we apply Theorem 5.7.31 of [1], we have

$$
q\left(A \circ B^{-1}\right) \geq q(A) \min _{1 \leq i \leq n} \beta_{i i}=0.07003 .
$$

If we apply Theorem 9 of [2], we have

$$
q\left(A \circ B^{-1}\right) \geq \frac{1-\rho\left(J_{A}\right) \rho\left(J_{B}\right)}{1+\rho^{2}\left(J_{B}\right)} \min _{1 \leq i \leq n} \frac{a_{i i}}{b_{i i}}=0.05229 .
$$

If we apply Theorem 2.1 of [10], we have

$$
q\left(A \circ B^{-1}\right) \geq \min _{1 \leq i \leq n}\left\{\frac{a_{i i}-s_{i} \sum_{j \neq i}\left|a_{j i}\right|}{b_{i i}}\right\}=0.08
$$

But if we apply Theorem 2.4, we have

$$
q\left(A \circ B^{-1}\right) \geq\left(1-\rho\left(J_{A}\right) \rho\left(J_{B}\right)\right) \min _{1 \leq i \leq n} \frac{a_{i i}}{b_{i i}}=0.08291 .
$$

In fact, $q\left(A \circ B^{-1}\right)=0.21478$. Example 2.1 shows that the bound in (2.6) is better than these corresponding bounds in $[1,2,10]$.

\section{Competing interests}

The authors declare that they have no competing interests.

Authors' contributions

All authors conceived of the study, participated in its design and coordination, drafted the manuscript, participated in the sequence alignment, and read and approved the final manuscript.

\section{Author details}

${ }^{1}$ Department of Mathematics, East China Normal University, Shanghai, 200241, People's Republic of China. ${ }^{2}$ Department of Mathematics, Northeast Forestry University, Harbin, 150040, People's Republic of China. 


\section{Acknowledgements}

The work of DZ is supported by the Scholarship Award for Excellent Doctoral Students granted by East China Normal University (No. XRZZ2012021). The work of GC is supported by the National Natural Science Foundation of China (No. 11071079) and the Natural Science Foundation of Zhejiang Province (No. Y6110043). The work of XZ is supported by the National Natural Science Foundation of China (No. 10901056) and the Science and Technology Commission of Shanghai Municipality (No. 11QA1402200).

Received: 14 September 2012 Accepted: 21 December 2012 Published: 14 January 2013

\section{References}

1. Horn, RA, Johnson, CR: Topics in Matrix Analysis. Cambridge University Press, Cambridge (1991)

2. Huang, R: Some inequalities for the Hadamard product and the Fan product of matrices. Linear Algebra Appl. 428, 1551-1559 (2008)

3. Fiedler, M, Johnson, CR, Markham, T, Neumann, M: A trace inequality for M-matrices and the symmetrizability of a real matrix by a positive diagonal matrix. Linear Algebra Appl. 71, 81-94 (1985)

4. Fiedler, M, Markham, T: An inequality for the Hadamard product of an M-matrix and inverse M-matrix. Linear Algebra Appl. 101, 1-8 (1988)

5. Chen, SC: A lower bound for the minimum eigenvalue of the Hadamard product of matrices. Linear Algebra Appl. 378, 159-166 (2004)

6. Xiang, $\mathrm{SH}$ : On an inequality for the Hadamard product of an $\mathrm{M}$-matrix or an $\mathrm{H}$-matrix and its inverse. Linear Algebra Appl. 367, 17-27 (2003)

7. Brauer, A: Limits for the characteristic roots of a matrix II. Duke Math. J. 14, 21-26 (1947)

8. Yong, XR, Wang, Z: On a conjecture of Fiedler and Markham. Linear Algebra Appl. 288, 259-267 (1999)

9. Berman, A, Plemmons, RJ: Nonnegative Matrices in the Mathematical Sciences. SIAM, Philadelphia (1994)

10. Li, YT, Li, YY, Wang, RW, Wang, YQ: Some new bounds on eigenvalues of the Hadamard product and the Fan product of matrices. Linear Algebra Appl. 432, 536-545 (2010)

doi:10.1186/1029-242X-2013-16

Cite this article as: Zhou et al.: Some inequalities for the Hadamard product of an M-matrix and an inverse M-matrix. Journal of Inequalities and Applications 2013 2013:16.

\section{Submit your manuscript to a SpringerOpen ${ }^{\circ}$ journal and benefit from:}

- Convenient online submission

- Rigorous peer review

- Immediate publication on acceptance

Open access: articles freely available online

- High visibility within the field

- Retaining the copyright to your article 